San Jose State University

From the SelectedWorks of Santosh KC

January 9, 2014

\title{
Electronic properties of InP (001)/HfO2 (001) interface: Band offsets and oxygen dependence
}

Santosh KC, San Jose State University 


\title{
Electronic properties of $\operatorname{lnP}(001) / \mathrm{HfO}_{2}(001)$ interface: Band offsets and oxygen dependence
}

\author{
Santosh KC, ${ }^{1}$ Hong Dong, ${ }^{1}$ Roberto C. Longo, ${ }_{1}^{1}$ Weichao Wang, ${ }^{2}$ Ka Xiong, ${ }^{1}$ \\ Robert M. Wallace, ${ }^{1,3}$ and Kyeongjae Cho ${ }^{1,3, a)}$ \\ ${ }^{1}$ Department of Materials Science \& Engineering, The University of Texas at Dallas, Richardson, \\ Texas 75080, USA \\ ${ }^{2}$ Department of Electronics \& Microelectronics and Tianjin Key Laboratory of Photo-Electronic Thin Film \\ Device and Technology, Nankai University, Tianjin 300071, China \\ ${ }^{3}$ Department of Physics, The University of Texas at Dallas, Richardson, Texas 75080, USA
}

(Received 25 October 2013; accepted 18 December 2013; published online 9 January 2014)

Using ab-initio methods, atomic structures and electronic properties of $\mathrm{InP}(001) / \mathrm{HfO}_{2}(001)$ interface are studied within the framework of density functional theory. We examine the $\mathrm{InP} / \mathrm{HfO}_{2}$ model interface electronic structures under varying oxidation conditions. The effects of indium and phosphorous concentrations on interfacial bonding, defect states, band offsets, and the thermodynamic stability at the interface are also investigated. The origin of interfacial gap states in $\mathrm{InP}(001) / \mathrm{HfO}_{2}$ (001) interface are proposed, mainly from the P-rich oxides, which is validated by our experimental work. This highlights the importance of surface passivation prior to high-K deposition based on the in situ spectroscopic results of atomic layer deposition of $\mathrm{HfO}_{2}$ on InP. (C) 2014 AIP Publishing LLC. [http://dx.doi.org/10.1063/1.4861177]

\section{INTRODUCTION}

To overcome the scaling limit of Si transitors, great attention has been recently turned to the zinc-blend III-V compound semiconductors for application in the metaloxide-semiconductor field-effect transistor (MOSFET) due to their superior electron mobility and higher breakdown field compared to $\mathrm{Si}^{1}{ }^{1,2}$ There have been significant research efforts in order to produce stable and high quality gatedielectric interfaces based on III-V semiconductors. ${ }^{2-4}$ However, the poor interface quality between the III-V channel and gate oxides has hindered their electrical performance. ${ }^{2,5,6}$ The interface defects between III-V and dielectric (high-к) oxides might induce the harmful Fermi level pinning. ${ }^{7-9}$ Improving the interface quality between high-K oxides and III-V compounds is a great challenge in achieving better device performances. Substantial efforts are currently undertaken focusing on understanding the origin of the interfacial defects and possible methods to overcome them using various passivation techniques. ${ }^{6,10,11}$

InP has a potential to be used as a barrier layer between high-K oxides and InGaAs high mobility channel for III-V semicondutor based quantum well FETs (QWFETs). ${ }^{12}$ The barrier layer is believed to minimize the defects introduced due to the oxidation of the channel materials. The InP/high- $\mathrm{K}$ oxide interface quality impacts the "off-state" performance of semiconductor devices such as sub-threshold swing (SS). ${ }^{13}$

Recently, several studies have reported that the surface oxidation of the III-V surface ${ }^{14-16}$ induces surface defect states. ${ }^{17} \mathrm{~A}$ detailed theoretical understanding of the origin of gap states of $\mathrm{GaAs} / \mathrm{HfO} \mathrm{O}_{2}$ and $\mathrm{GaSb} / \mathrm{HfO}_{2}$ interfaces and their possible passivation have also been studied previously. ${ }^{6,18}$ The interface states are mainly due to interfacial defects

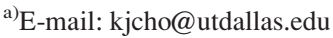

(atoms with mismached valance charge or bond configuration) rather than due to intrinsic gap states like the metal induced gap states (MIGS). ${ }^{19}$ However, a detailed theoretical study on the InP/ $/ \mathrm{HfO}_{2}$ interface, to identify the origin of the defects is not yet available. A detailed study of the atomic and electronic structrures of this III-V/High-K interface provides insight on the origin of the interface defect states. ${ }^{18-22}$

In this paper, an investigation of the origin of interface gap states of an ideal $\mathrm{InP}(001) / \mathrm{HfO}(001)$ interface and its dependence on the amount of interfacial oxygen concentration are presented. In addition, we also explain the effects of interfacial indium and phosphorous contents on the interfacial bonding, band offsets, and the thermodynamic stability of the interface. Moreover, we correlate our study with the experimental work on atomic layer deposition (ALD) of $\mathrm{HfO}_{2}$ on a native oxide InP (001) surface.

\section{METHODOLOGY}

First-prinicples calculations have been performed within the framework of Density Functional Theory (DFT) $)^{23-26}$ with plane wave basis set and Projector Augmented Wave $(\mathrm{PAW})^{27,28}$ pseudopotentials, as implemented in the Vienna Ab-initio Simulation Package (VASP)..$^{23,29-31}$ The electronic wave functions are represented by plane wave basis with a cutoff energy of $450 \mathrm{eV}$. The exchange correlation interactions are described within the Generalized Gradient Approximation (GGA). ${ }^{32}$

To investigate the $\mathrm{InP} / \mathrm{HfO}_{2}$ interface, an interface model has been constructed starting with In terminated InP (001) surface and $\mathrm{O}$ terminated c- $\mathrm{HfO}_{2}(001)$ surface with a lattice mismatch of $3.3 \%$ as shown in Figure 1. Since the interface electronic structure depends mainly on the local bonding configurations rather than the long range crystal order, the interface physics will be applicable to all phases of $\mathrm{HfO}_{2}{ }^{6}{ }^{6}$ This interface model contains 10 atomic layers of In 


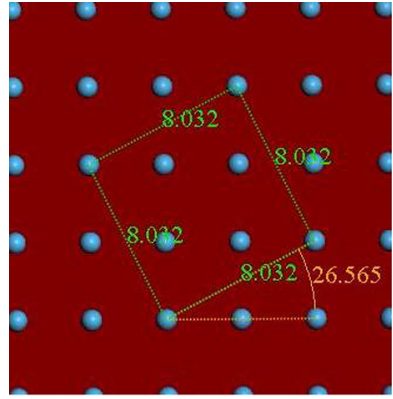

(a)

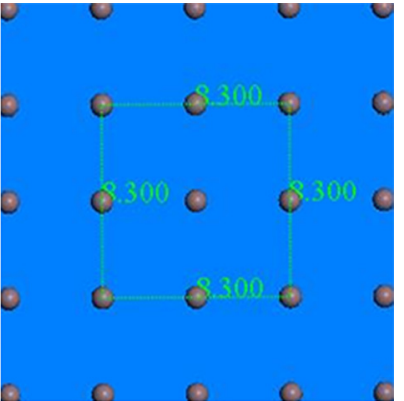

(b)
FIG. 1. (a) Hf monolayer of $\mathrm{HfO}_{2}$ (001), and (b) In monolayer of InP (001) bulk, along the normal to (001) direction. Interface supercell units are indicated as squares.

and 9 atomic layers of $\mathrm{P}$ (19 layers of InP) and 5 atomic layers of $\mathrm{Hf}$ and 6 atomic layers of $\mathrm{O}$ for a total of 164 atoms, to minimize the quantum size effect. Periodically repeated slabs are used to model the interface. Each periodic slab is separated by $10 \AA$ vacuum to avoid periodic image interaction between the two surfaces of the slab. To guarantee that all the gap states are originated only from interfacial atomic interactions, the top and bottom surfaces are passivated. The In terminated bottom layer is passivated by pseudo-hydrogen (with charge $1.25 \mathrm{e}$ ) atoms to mimic phosphorous bulk bonds. Half of the top oxygen atoms are removed to ensure an insulating $\mathrm{HfO}_{2}$ top surface. In our calculations, the atoms are allowed to relax, while the cell size is kept fixed. A gamma centered $4 \times 4 \times 1 \mathrm{k}$-point mesh is used in the self-consistent field (SCF) calculations, and a $8 \times 8 \times 1 \mathrm{k}$-point mesh is used for density of states (DOS) calculations. SCF dipole correction implemented in the VASP code is used to cancel possible spurious electric fields that may be induced by the periodic boundary conditions. The tolerance in the energy and forces was $10^{-4} \mathrm{eV}$ and $0.01 \mathrm{eV} / \AA$, respectively.

The DFT results are compared to interfacial chemistry studies of the $\mathrm{HfO}_{2} / \mathrm{InP}$ in which twenty cycles of ALD of $\mathrm{HfO}_{2}$ on a native oxide InP (001) surface has been carried out at $300{ }^{\circ} \mathrm{C}$. The ALD reactor and the in situ monochromatic $(\mathrm{h} v=1486.7 \mathrm{eV}) \mathrm{X}$-ray photoelectron spectroscopy (XPS) system are integrated by an UHV transfer tube, allowing the samples transferring between ALD and XPS chamber without exposure to atmosphere. The details of the experimental system are described elsewhere. ${ }^{10}$ Tetrakis (dimethylamino) hafnium (TDMA-Hf) is used as the metal precursor and de-ionized water vapor is used an oxidant precursor with high purity $\mathrm{N}_{2}$ gas as the carrier gas (with a gas flow of 200 SCCM, with the pressure in the ALD chamber at 10 mbars during ALD). The TDMA-Hf vessel is maintained at $90^{\circ} \mathrm{C}$, and delivered by heated lines/valves at $135^{\circ} \mathrm{C}$. Both precursors underwent $0.1 \mathrm{~s} / 20 \mathrm{~s}$ for precursor pulse time/purging time for each precursor. The substrate is maintained at a temperature of $300{ }^{\circ} \mathrm{C}$ during ALD. One n-type (S-doped $10^{18} \mathrm{~cm}^{-3}$ ) InP (100) sample is used, in this study. The sample was degreased in acetone, methanol, and isopropanol for $1 \mathrm{~min}$ each at room temperature. The XPS scans are taken at $45^{\circ}$ respect to the sample surface normal before and after the 20 cycles of $\mathrm{HfO}_{2}$ ALD. The detailed XPS fitting processes are described elsewhere. ${ }^{10}$

\section{RESULTS AND DISCUSSION}

\section{A. Interfacial bonding}

First, the local atomic interaction in the InP (001) $/ \mathrm{HfO}_{2}(001)$ interface model is investigated. The bonding information is crucial to understand the gap states generated by unsaturated bonds at the interface. Upon structure optimization of the interface, the interfacial In is shifted from the InP region (accompanying In-P bond breaking) towards the $\mathrm{HfO}_{2}$ domain to form an In-O bond, which is shown in Figure 2. As a result, unsaturated $\mathrm{P}$ atoms may reconstruct themselves to form $\mathrm{P}$ dimer or POx depending on the oxygen concentration at the interface. Thus, the formation of $\mathrm{InO}_{\mathrm{x}}, \mathrm{PO}_{\mathrm{x}}$, and $\mathrm{P}$ dimers is observed at the interface for an ideal interface.

Since the ideal cleaved $\mathrm{HfO}_{2}(001)$ surface initially has 10 oxygen atoms (for the model supercell used in the study), we systematically decreased the number of interfacial $\mathrm{O}$ atoms from 10 (100\% of interfacial oxygen) to three (30\% of interfacial oxygen) in order to simulate the relevant ALD growth conditions at different oxygen partial pressures. The Bader charges ${ }^{33-35}$ of the interfacial atoms are calculated and compared with the corresponding bulk values. The Bader charges and the corresponding bonding information are shown in Table I. The table shows all in-equivalent interfacial atomic charges and the interfacial bond lengths. Interfacial In (with one dangling bond) has a charge excess, and $\mathrm{P}$ has a charge deficit with respect to the bulk values. The Indium charge at the interface depends on its coordination: for 2-fold $(2 \mathrm{O})$ is $2.32 \mathrm{e}, 5$-fold $(3 \mathrm{O}, 2 \mathrm{P})$ is $1.63 \mathrm{e}$, and 5 -fold $(4 \mathrm{O}, 1 \mathrm{P})$ is $1.69 \mathrm{e}$. Similarly, $\mathrm{P}$ at the interface has an electronic charge of $2.94 \mathrm{e}$ for 4 -fold (4O). This interface is much more complex than that of conventional $\mathrm{Si} / \mathrm{SiO}_{2}$ interface, ${ }^{21}$ due to the different natures of bonding at the interface. Moreover, bulk InP has polar covalent bonding, $\mathrm{HfO}_{2}$ has ionic bonding, In-O has ionic bonding, and P-O is a polar covalent bond. Thus, bonding saturation at the interface is
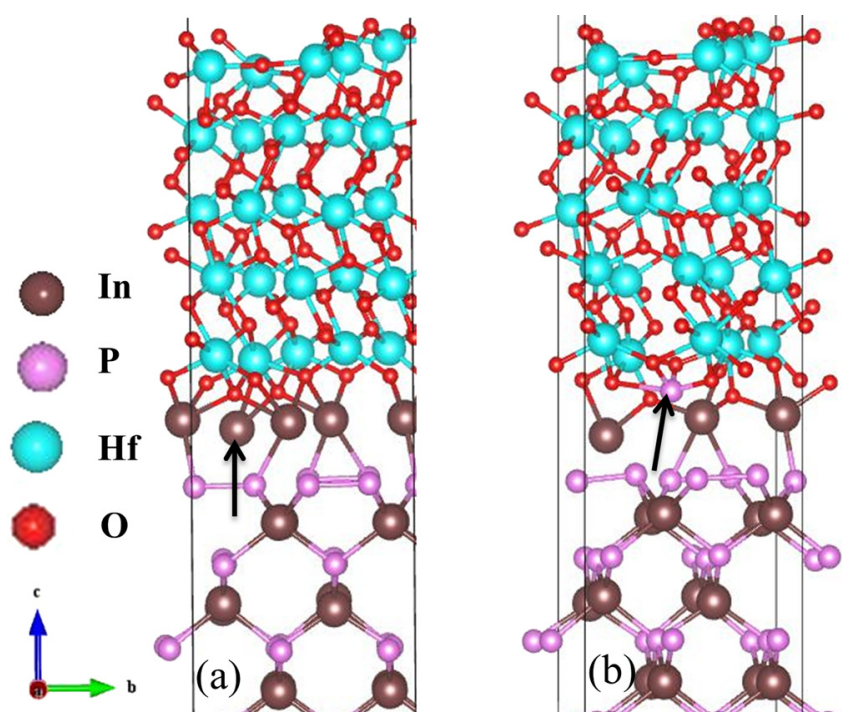

FIG. 2. Side view of $\mathrm{InP} / \mathrm{HfO}_{2}$ interfacial bonding-(a) $\mathrm{O}_{10}$ model and (b) $\mathrm{O}_{10-1 \mathrm{P}}$ model. In, $\mathrm{P}, \mathrm{Hf}, \mathrm{O}$ atoms are represented by brown, pink, green, and red balls, respectively. 
TABLE I. Bader charges and interfacial bonding distances at the InP $(001) / \mathrm{HfO}_{2}(001)$ interface.

\begin{tabular}{lccc}
\hline \hline Model & In interface & O Interface & In-O bond $(\AA)$ \\
\hline $\mathrm{O}_{10}$ & $1.53 \mathrm{e}$ & $7.90 \mathrm{e}$ & $2.07,2.12,2.16,2.18,2.27$ \\
$\mathrm{O}_{9}$ & $1.65 \mathrm{e}$ & $8.02 \mathrm{e}$ & $2.12,2.22,2.23,2.49,2.44$ \\
$\mathrm{O}_{8}$ & $1.63 \mathrm{e}$ & $8.02 \mathrm{e}$ & $2.23,2.29,2.34,2.56,2.64$ \\
$\mathrm{O}_{7}$ & $1.81,1.46,1.87 \mathrm{e}$ & $8.01 \mathrm{e}$ & $2.29,2.30,2.36,2.44,2.61$ \\
$\mathrm{O}_{6}$ & $2.04,1.6,2.46,2.24 \mathrm{e}$ & $7.99,7.83,7.81 \mathrm{e}$ & $2.41,2.43,2.44,2.48$ \\
$\mathrm{O}_{5}$ & $2.8,2.44,2.5 \mathrm{e}$ & $7.73,7.84 \mathrm{e}$ & $2.37,2.70,2.59,2.60$ \\
$\mathrm{O}_{4}$ & $2.56,2.49,2.94 \mathrm{e}$ & $7.76 \mathrm{e}$ & $2.41,2.44$ \\
$\mathrm{O}_{3}$ & $2.87,2.56,2.36 \mathrm{e}$ & $7.71 \mathrm{e}$ & $2.65,2.93$ \\
\hline \hline
\end{tabular}

something very difficult to achieve, and the resulting partially saturated bonds induce interfacial gap states.

\section{B. Interface defect states}

In a FET, the gate field can sweep the Fermi level across the semiconductor (III-V) band gap to vary the carrier density in the channel. However, the III-V/high-K interfaces are known to have large interface density of states within the band gap pinning the Fermi level, resulting in a poor device performance. This is a major challenging issue in III-V based device realization, and to overcome this problem, an understanding of the origin of defect states at the atomic level is needed.

Figure 3 shows that electronic structures of the $\mathrm{InP} / \mathrm{HfO}_{2}$ interfaces with several levels of oxygen concentrations, from $100 \%$ to $30 \%\left(\mathrm{O}_{10}-\mathrm{O}_{3}\right)$ and corresponding partial charge densities for the defect states are shown in Figure 4. Similarly, electronic structures of the $\mathrm{InP} / \mathrm{HfO}_{2}$ interfaces with various $\mathrm{P}$ content at the interface and their corresponding partial charge densities for the interface system are shown in Figures 5 and 6, respectively. The dotted region in the electronic band structure represents the contribution from the bulk InP, whereas the lines without dots represent the bands associated purely with the interface. The GGA bulk InP band gap is approximately $1 \mathrm{eV}$. The band structure for an $\mathrm{O}_{10}$ interface model shows gap states within the bulk band gap of InP, thus, causing Fermi level pinning. The partial charge density (see Fig. 4) reveals that the flat band gap states originate from a $\mathrm{P}$ dimer and an In dangling bond. Similarly, we observed the defect states for lower interfacial oxygen concentration. At a very low oxygen concentration (see Fig. $4 . \mathrm{O}_{3}$ model), the formation of $\mathrm{Hf}$ dangling bonds is expected, which may increase the defect concentration. However, the formation of these species is not realistic experimentally, given the relative $\mathrm{O}$ partial pressure during typical ALD conditions.
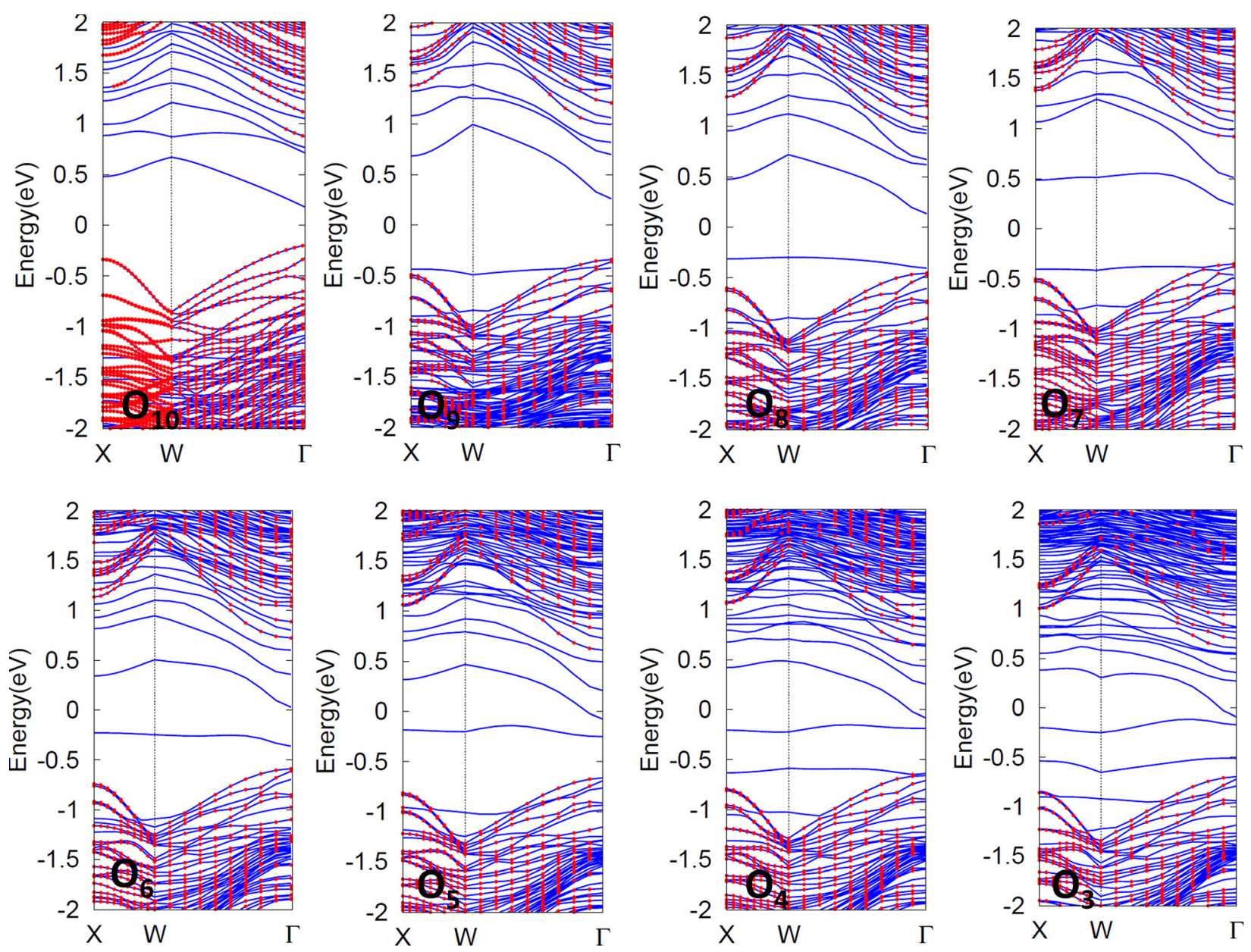

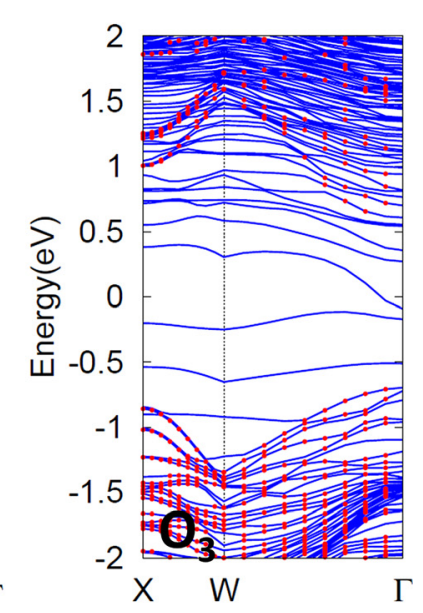

FIG. 3. Band structure for the various interface configurations plotted with the projected InP bulk band structure. The dotted region indicates the projected InP bulk bands. The high symmetry k-points $\mathrm{X}, \mathrm{W}, \Gamma$ are at $(0.5,0,0),(0.5,0.5,0),(0,0,0)$, respectively. The Fermi level is set to zero of the energy axis. 


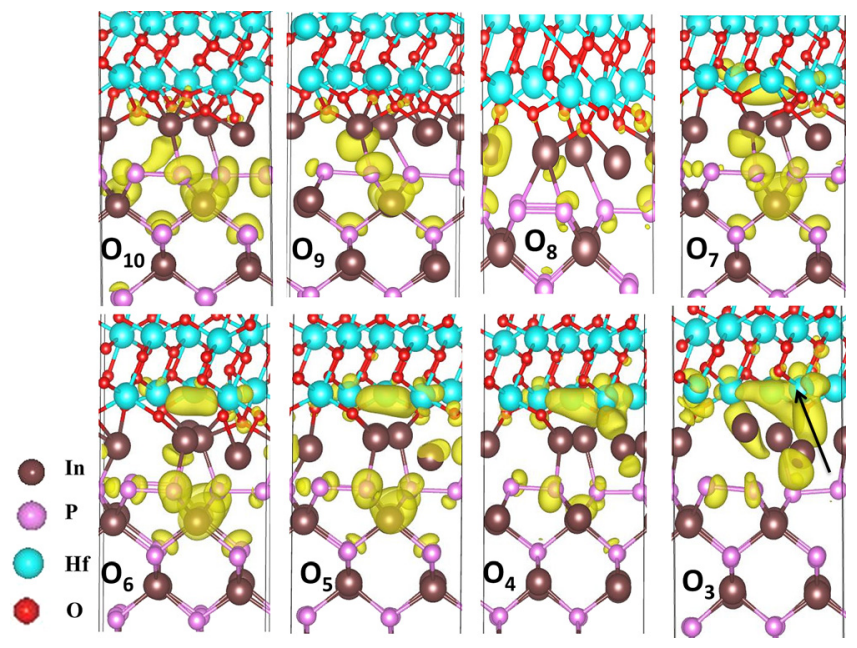

FIG. 4. Partial charge density distributions for the gap states. In and $\mathrm{P}$ atoms are indicated by brown, pink spheres, respectively. Yellow color in the figure indicates the partial charge distribution. Isosurfaces shown in the figure correspond to the isovalues of $2 \times 10^{-3} \mathrm{e}^{\circ}$.
It is observed that interfacial In-P bonds are broken to form oxides at the ideal interface. As a consequence, a $\mathrm{P}$ dimer is likely to be formed, which is attributed to an interfacial charge transfer in order to compensate charge loss with respect to the bulk electronic structure. (Note that a similar effect has been obtained for the GaAs: $\mathrm{HfO}_{2}$ interface. ${ }^{6}$ ) As the oxygen concentration is lowered, the defect density increases. At high oxygen concentration at the interface, the gap states originate from $\mathrm{InO}_{\mathrm{x}}$ and $\mathrm{PO}_{\mathrm{x}}$ bonding. The defect states are then due to the unsaturated oxides of indium or phosphorous for an oxygen rich condition.

The interface defect trap density $\left(D_{i t}\right)$ increases as the unsaturated $\mathrm{PO}_{\mathrm{x}}$ species are present at the interface (see Fig. 7) consistent with the experimental study. ${ }^{5}$ More specifically, $D_{i t}$ increases in the $\mathrm{O}_{10-2 \mathrm{P}}, \mathrm{O}_{10-3 \mathrm{P}}$, and $\mathrm{O}_{10-4 \mathrm{P}}$ models as compared to the $\mathrm{O}_{10}$ model. Defect trap densities have been correlated to the $\mathrm{PO}_{\mathrm{x}}$ in recent experimental studies. ${ }^{5,10,36}$ Recent study has also shown that In out-diffusion of the InP phase with an undetectable $\mathrm{P}$ out-diffusion upon post deposition annealing of high- $\kappa$ results in unsaturated phosphorous oxides and, as a consequence, a large $D_{i t}$ is observed. ${ }^{5,37}$ Recent study on InGaAs also shows significant In diffusion
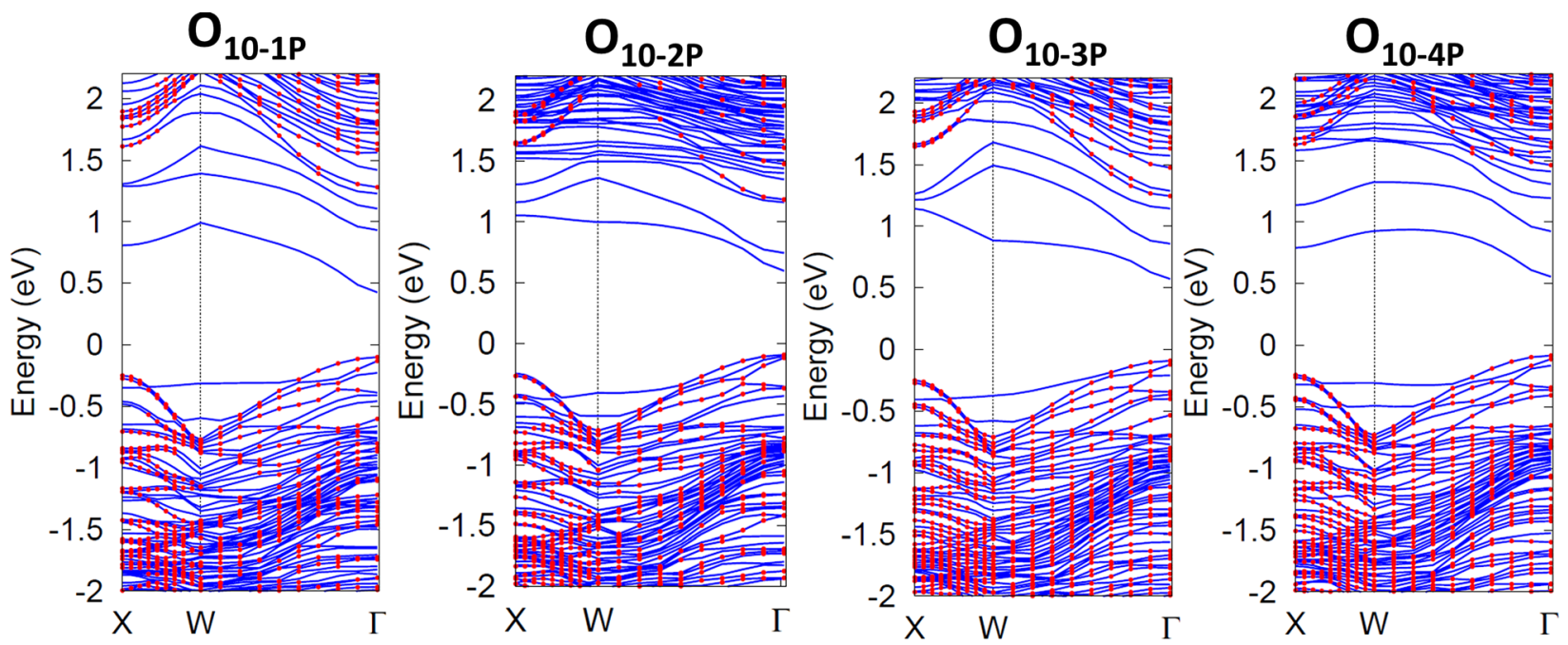

FIG. 5. Band structure for the various In/P concentrations at the interface plotted with the projected InP bulk band structure. The dotted region indicates the projected InP bulk bands. The high symmetry k-points $\mathrm{X}, \mathrm{W}, \Gamma$ are at $(0.5,0,0),(0.5,0.5,0),(0,0,0)$, respectively. The Fermi level is set to zero of the energy axis.

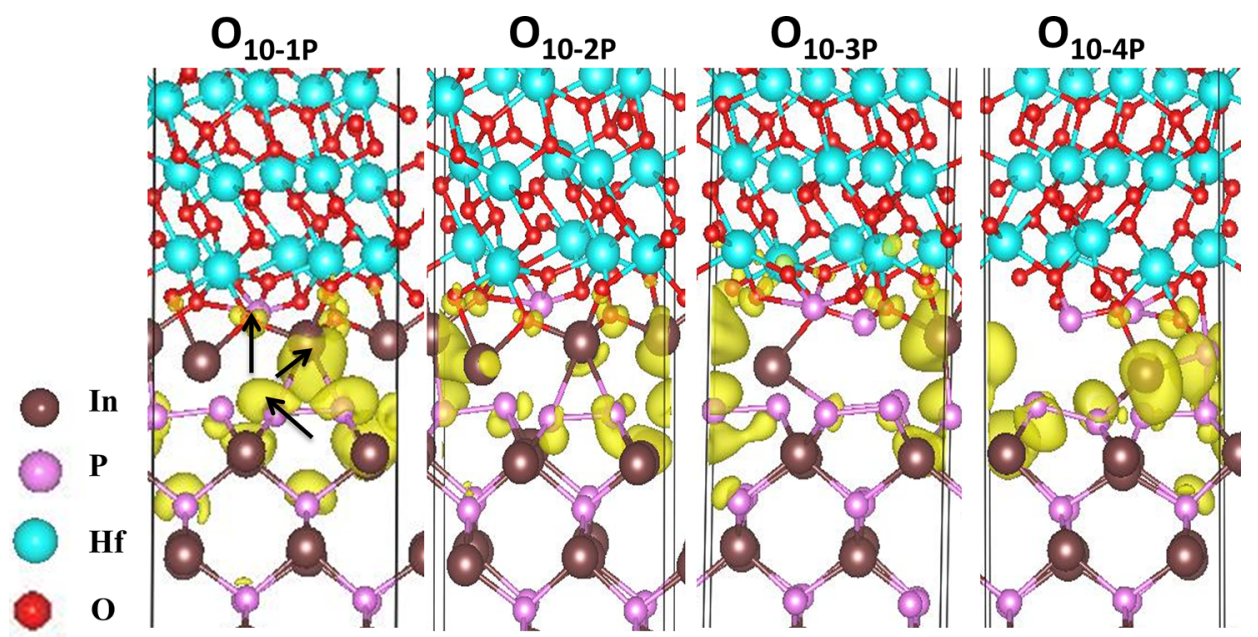

FIG. 6. Partial charge density distributions for the gap states. In and P atoms are indicated by brown, pink spheres, respectively. Yellow color in the figure indicates the partial charge distribution. Isosurfaces shown in the figure correspond to the isovalues of $2 \times 10^{-3}$ e $\AA^{-3}$. 

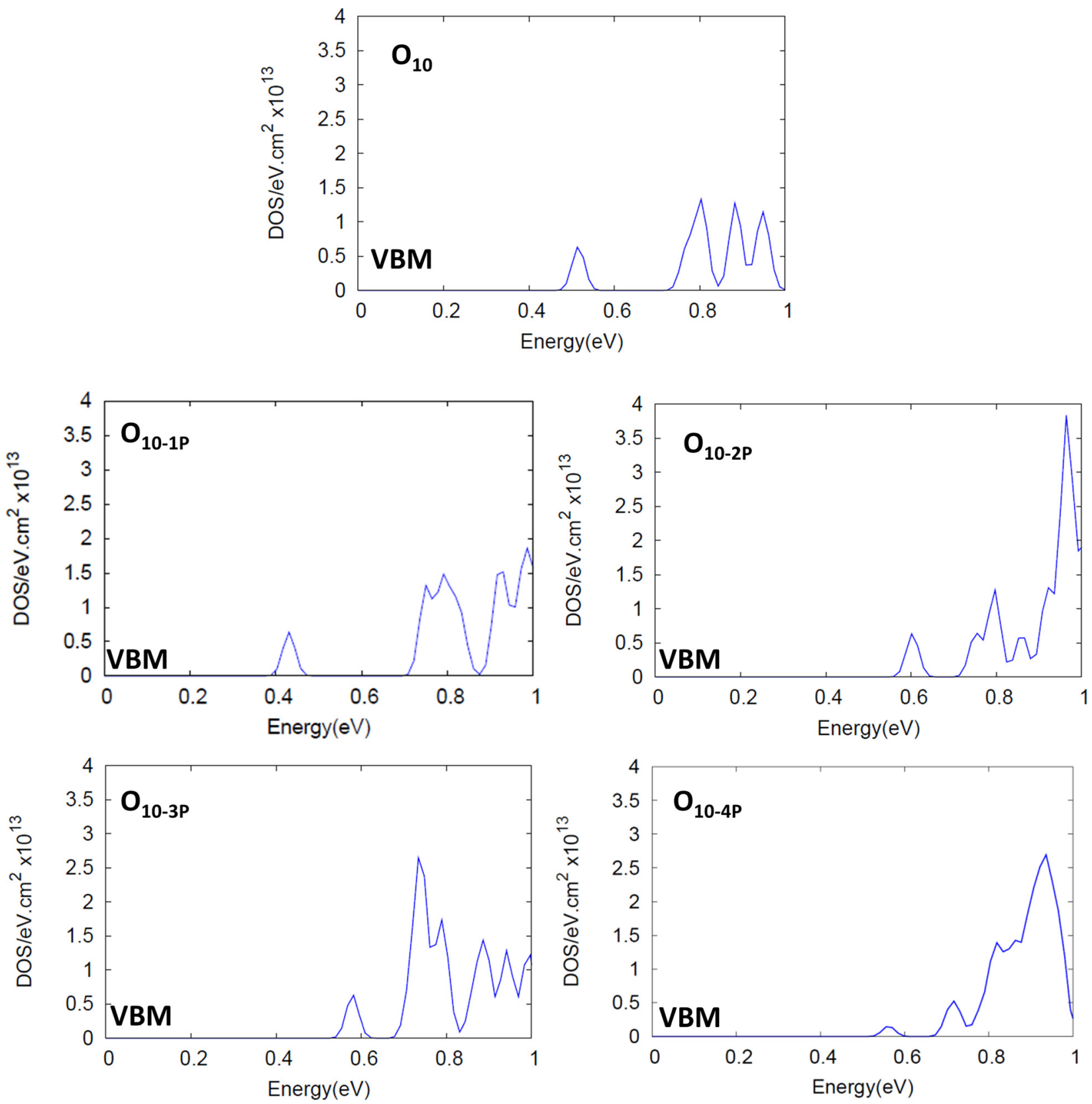

FIG. 7. Defect trap density $\left(\mathrm{D}_{\mathrm{it}}\right)$ computed for the $\mathrm{InP} / \mathrm{HfO}_{2}$ interface model with different $\mathrm{P}$ contents at the interface.

across the high- $\kappa$ dielectrics. ${ }^{38}$ This questions the thermal stability and reliability issues of the III-V semiconductor device. Thus, more research is needed to focus on controlling the defects during ALD growth and to find suitable passivation techniques and/or species in order to minimize the defect trap density.

Figures 8 (a) and 8 (b) show the P $2 p$ and In $3 d_{5 / 2}$ core level XPS spectra, respectively, before and after a 20 cycle ALD $\mathrm{HfO}_{2}$ growth process. In-oxide concentrations are seen to decrease significantly due to the "self-cleaning" effect of the ALD precursor on the In-oxides. However, the $\mathrm{P}$-oxides concentration maintains a similar level before and after the ALD process as shown in Figure 8. This persistent P-oxide concentration during the ALD process and the correlation of interfacial band gap states suggest that it is extremely important to passivate the unsaturated surface bonds prior to ALD to achieve the suitable device performance. ${ }^{39}$

\section{Band offsets between InP and $\mathrm{HfO}_{2}$}

The oxide-semiconductor band offset has been calculated as a function of the interface oxygen concentration. The band offset has strong effects on the quantum mechanical electron tunneling mechanism across the interface. It is known that the conduction band offset $(\mathrm{CBO})$ is, in general, smaller than the valence band offset (VBO) in III-V semiconductors. ${ }^{40}$ Thus, the band offsets are important quantities that need to be considered for controlling the device leakage. The band offset between InP and $\mathrm{HfO}_{2}$ is related to the interfacial dipole induced by charge transfer. In the absence of charge transfer, the $\mathrm{BO}$ would be directly obtained through 


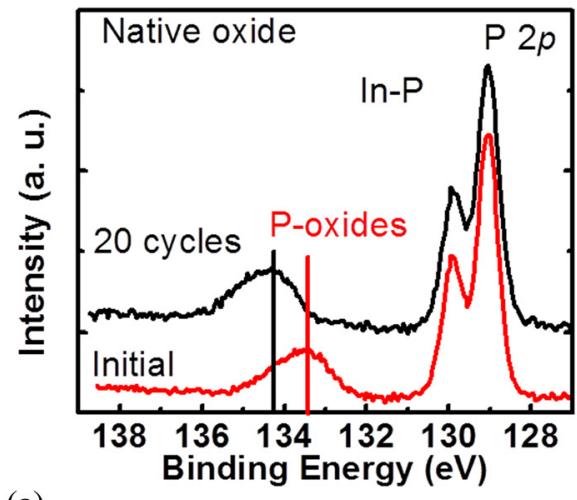

(a)

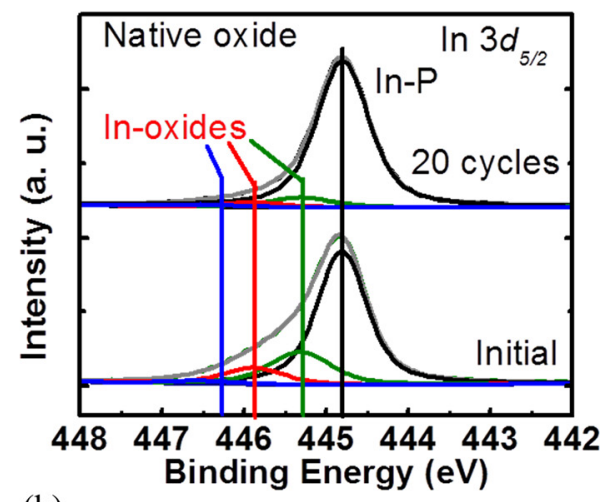

(b)
FIG. 8. (a) P $2 p$ and (b) In $3 d 5 / 2$ core level XPS spectra for initial and 20 cycles of ALD $\mathrm{HfO}_{2}$ deposited on native oxide $\mathrm{InP}(100)$ surface at $300^{\circ} \mathrm{C}$. the electron affinity (EA) rule. In the models employed here, the interfacial oxygen content is varied, which directly affects both the charge transfer and the electron affinity. A schematic diagram of band offsets between $\mathrm{InP}$ and $\mathrm{HfO}_{2}$ is shown in Figure 9. Since, hybrid energy functional (HSE) study on GaAs/high-K or GaSb/high-к did not change the qualitative conclusion of the study apart from slight improvement in band offsets, ${ }^{6,18}$ present study is carried out at GGA level of approximation.

Since, valance bands (VBs) are very accurately computed from DFT than the conduction bands, VBO is obtained analyzing the local DOS (LDOS) of bulk region of InP and $\mathrm{HfO}_{2}$. The LDOS of atoms in the InP and $\mathrm{HfO}_{2}$ layers away from the interface region are computed and the obtained energy difference between their valence band maxima (VBM) is shown in Table II. The CBO can then be estimated by using the experimental band gaps of $\mathrm{InP}$ and $\mathrm{HfO}_{2}$.

The change in the $\mathrm{O}$ content at the interface affects the states of $\mathrm{HfO}_{2}$, but not those of the InP, since the VBM of $\mathrm{HfO}_{2}$ is dominated by $\mathrm{O} 2 \mathrm{P}$ states. The computaional results show that reduced $\mathrm{O}$ content shifts the $\mathrm{VB}$ edge of $\mathrm{HfO}_{2}$ down due to the reduction of the $\mathrm{O} 2 \mathrm{P}$ distribution at the VBM. As a consequence, the VBO increases with the reduction of the amount of interfacial oxygen. Thus, VBO can

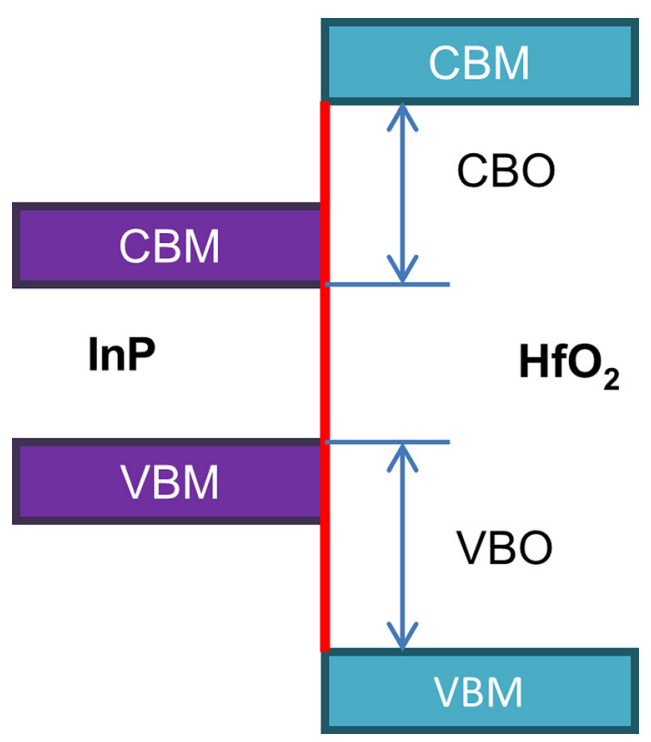

FIG. 9. Schematic diagram of band offsets between InP and $\mathrm{HfO}_{2}$. experimentally be controlled by tuning the oxygen concentration, a property directly related to the ambient oxygen pressure. By gradually reducing the interfacial $\mathrm{O}$ content from $100 \%$ to $30 \%$, the VBO can be modified up to $1.8 \mathrm{eV}$. Our data are comparable to the previously reported values for $\mathrm{CBO}$ and $\mathrm{VBO}$ of $1.74 \mathrm{eV}$ and $2.67-2.9 \mathrm{eV}$, respectively, for other III-V/ $/ \mathrm{HfO}_{2}$ systems. $^{40}$

\section{Interface formation energy}

In order to determine the interface stability of different interface models, the formation energies of each model are investigated. The ground state of the interface model is determined by minimizing the thermodynamic potential energy. The relative stability of the interface model is then obtained by comparing their formation energies, given by Eq. (1),

$$
E^{f o r m}=\frac{E_{t o t}-n E_{H f}-k \mu_{0}-m E_{I n}-p E_{P}}{A},
$$

where $E^{\text {form }}$ is the interface formation energy, $E_{t o t}$ is the total energy of the system, $A$ is the total interface area of the model slab, and $\mu_{i}$ are the chemical potentials for the respective elements with $n, m, k$, and $p$ being numbers of $\mathrm{Hf}$, In, O, and $\mathrm{P}$ atoms, respectively. The total DFT energies are used instead of the Gibb's free energy for the calculations of the formation energies since the entropy contributions and enthalpy changes due to finite temperatures (e.g., at $300-600 \mathrm{~K}$ ) are insignificant for all of the structures,

TABLE II. Band offsets between $\mathrm{InP}$ and $\mathrm{HfO}_{2}$ for various interfacial oxygen contents.

\begin{tabular}{lc}
\hline \hline Interface model & VBO $(\mathrm{eV})$ \\
\hline $\mathrm{O}_{10}$ & 0.7 \\
$\mathrm{O}_{9}$ & 1.3 \\
$\mathrm{O}_{8}$ & 1.4 \\
$\mathrm{O}_{7}$ & 1.8 \\
$\mathrm{O}_{6}$ & 2.2 \\
$\mathrm{O}_{5}$ & 2.4 \\
$\mathrm{O}_{4}$ & 2.5 \\
$\mathrm{O}_{3}$ & 2.5 \\
$\mathrm{CNL}$ & $2.67-2.9 \mathrm{eV}$ (Ref. 40) \\
\hline \hline
\end{tabular}


resulting in an insignificant change in the relative interface formation energies. ${ }^{41,42}$

We can write the bulk $\mathrm{HfO}_{2}$ in terms of gas phase oxygen molecule and Hafnium metal,

$$
E_{H f O 2}(b u l k)=E_{H f}(b u l k)+\mu_{O 2}(b u l k)+\Delta H_{H f O 2}^{f} .
$$

Using Eq. (2) and setting the bulk oxygen as reference, the numerator of Eq. (1) can be re-written,

$E^{f o r m}=E_{t o t}-\left[n\left(E_{H f O 2}(\right.\right.$ bulk $\left.\left.)-\Delta H_{H f O 2}^{f}\right)+m E_{I n}+p E_{P}-l \mu_{O}\right]$,

where $l=2 n-k$.

Rearranging this equation to obtain the interface formation energy per unit area yields

$E^{f o r m}=\frac{E_{\text {tot }}-\left[n E_{\mathrm{HfO} 2}(\text { bulk })+m E_{I n}+p\left(E_{I n P}-E_{I n}\right)\right]+l \mu_{O}}{A}$.

The limits of the oxygen chemical potential are

$$
\mu_{O} \leq \mu_{O}(\text { bulk })=\frac{1}{2} E_{O 2} \quad \text { and } \quad \mu_{H f} \leq \mu_{H f}(\text { bulk })=E_{H f} .
$$

Thus, the thermodynamic range of oxygen chemical potential for the interface model is obtained,

$$
\begin{aligned}
& \frac{1}{2}\left(E_{O 2}+\Delta H^{f}\right) \mu_{O} \leq \frac{1}{2} E_{O 2} \\
& \text { which is }-10.39 \mathrm{eV} \leq \mu_{O} \leq-5.01 \mathrm{eV} .
\end{aligned}
$$

Thus, the interface formation energy as a function of oxygen chemical potential $\left(\mu_{\mathrm{o}}\right)$ is calculated, as shown in Figure 10. The right hand side is O-rich limit (the bulk $\mu_{\mathrm{o}}$ is set to zero), whereas the left hand side of the plot represents the O-poor limit. The formation energy increases gradually when the oxygen chemical potential changes from the O-rich limit to the O-deficient limit for $\mathrm{O}_{10}$ to $\mathrm{O}_{6}$ interface models. However, this trend is reversed for the $\mathrm{O}_{4}$ and $\mathrm{O}_{3}$ models. It is found that the oxygen rich environment is always thermodnamically favorable.

At the Hf- rich limit, $\mathrm{O}_{10}$ is at the top and $\mathrm{O}_{3}$ at the bottom in the formation energy plot. Thus, $\mathrm{O}_{3}$ is the most stable in this case and $\mathrm{O}_{10}-\mathrm{O}_{6}$ may not exist at this extreme limit. On the other hand, at O-rich limit, we found that $\mathrm{O}_{10}$ is the most stable, where $\mathrm{O}_{3}$ is at top and $\mathrm{O}_{10}$ lies at the bottom in the formation energy plot, whereas $\mathrm{O}_{3}$ and $\mathrm{O}_{4}$ may not exist at this extreme limit. For $-6.4 \mathrm{eV} \leq \mu_{\mathrm{O}} \leq-5.01 \mathrm{eV}, \mathrm{O}_{10}$ is the most stable configuration, for $-7.7 \mathrm{eV} \leq \mu_{\mathrm{O}} \leq-6.4 \mathrm{eV}, \mathrm{O}_{9}$ is the most stable, for $-9.4 \mathrm{eV} \leq \mu_{\mathrm{O}} \leq-7.7 \mathrm{eV}$, the $\mathrm{O}_{8}$ model is the most stable and, finally, for $-9.8 \mathrm{eV} \leq \mu_{\mathrm{O}} \leq-9.4 \mathrm{eV}$, the $\mathrm{O}_{6}$ model seems to be the most stable interface (see Fig. 10 with $\mathrm{O}$ rich limit is set to zero). Our results indicate that changing the interfacial $\mathrm{O}$ content, we can control the interface stability. Experimentally, the Hf rich limit is not the most suitable oxide growth condition due to the metallic nature of interface. To compare a realistic oxide growth condition,

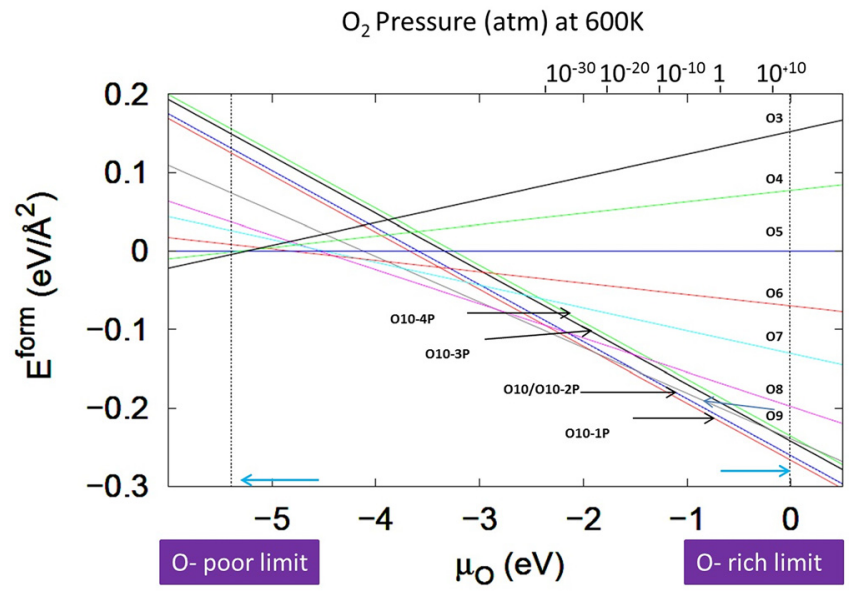

FIG. 10. Relative interface formation energy with respect to the oxygen chemical potential in the range: $-5.38 \mathrm{eV} \leq \mu_{\mathrm{O}} \leq 0 \mathrm{eV}$ (setting bulk value to zero).

TABLE III. Band offsets between InP and $\mathrm{HfO}_{2}$ for various interfacial $\mathrm{P}$ contents.

\begin{tabular}{ll}
\hline \hline Interface model & VBO $(\mathrm{eV})$ \\
\hline $\mathrm{O}_{10-1 \mathrm{P}}$ & 1.16 \\
$\mathrm{O}_{10-2 \mathrm{P}}$ & 2.15 \\
$\mathrm{O}_{10-3 \mathrm{P}}$ & 1.82 \\
$\mathrm{O}_{10-4 \mathrm{P}}$ & 2.24 \\
\hline \hline
\end{tabular}

ALD of $\mathrm{HfO}_{2}$ on $\mathrm{InP}$ at $600 \mathrm{~K}$ is used to correlate chemical potential with the oxygen partial pressure using Eq. (5),

$$
\mu_{0}(T, P)=\mu_{0}\left(T, P^{0}\right)+\frac{1}{2} k T \ln \left(\frac{p}{p^{0}}\right),
$$

where $P$ and $P^{O}$ are the pressures at $\mathrm{T}$ temperature and at one atmosphere, respectively. This provides the top axis in Figure 10 .

Changing the phosphorous content at the interface was also investigated to determine if such models are more favorable. It was found that models with $\mathrm{P}$ atoms at the interface are indeed energetically favorable. Among these models, the $\mathrm{O}_{10-1 \mathrm{P}}$ interface is the most stable (shown by red line), the $\mathrm{O}_{10-2 \mathrm{P}}$ model overlaps with $\mathrm{O}_{10}$ model (shown by blue- $\mathrm{O}_{10}$ ), $\mathrm{O}_{10-3 \mathrm{P}}$ (shown by black line) is less stable as compared to $\mathrm{O}_{10}$, $\mathrm{O}_{10-1 \mathrm{P}}$, and $\mathrm{O}_{10-2 \mathrm{P}}$ models, and $\mathrm{O}_{10-4 \mathrm{P}}$ is the least stable among all $\mathrm{O}_{10}$ models (green line). The corresponding band offsets for those interface models are also listed in Table III. The stability of such species is consistent with the persistent presence of the $\mathrm{PO}_{\mathrm{x}}$ at the interface by XPS, as shown above. ${ }^{37,43}$

\section{CONCLUSION}

In this work, a systematic investigation of the atomic and electronic structures of $\mathrm{InP} / \mathrm{HfO}_{2}$ interface is performed as a function of interfacial atomic species $(\mathrm{O}, \mathrm{In} / \mathrm{P})$ from $a b$ initio calculations. It is found that the interface formation energy changes with the $\mathrm{O}$ chemical potential, i.e., the $\mathrm{O}$ content. Gap states and flat bands are observed as a consequence of In dangling bonds and $\mathrm{P}$ dimers. Indium dimers are possible only for interfaces with low interfacial $\mathrm{O}$ 
content, whereas $\mathrm{Hf}$ dangling bonds and unsaturated $\mathrm{P}$ oxides increase defect population at low $\mathrm{O}$ content. Oxygen-rich conditions are favorable, whereas Hf-rich models are not realistic for ALD high- $\kappa$ field effect devices. The $\mathrm{O}_{10-1 \mathrm{P}}$ is the most stable among all the models considered here. At high oxygen content, the gap states originate from $\mathrm{InO}_{\mathrm{x}}, \mathrm{PO}_{\mathrm{x}}$, and $\mathrm{P}$ dimer species. It is also observed that the defect population increases with $\mathrm{P}$ content at the interface since it results in unsaturated P-oxides, which suggests the atomistic mechanism of interfacial defect generation at $\mathrm{InP} / \mathrm{HfO}_{2}$ interface due to $\mathrm{PO}_{\mathrm{x}}$ and is agreeing with the experimental findings.

\section{ACKNOWLEDGMENTS}

We thank all the members of III-V group at UTD for fruitful discussions, in particular, Professor Christopher Hinkle, Professor Yves Chabal, Dr. Barry Brennan, Dr. Rohit Galatage, and Wilfredo Cabrera. This work was supported by the Semiconductor Research Corporation FCRP Materials Structures and Devices (MSD) Center the Nanoelectronics Research Initiative and the National Institute of Standards and Technology (NIST) through the Midwest Institute for Nanoelectronics 125 Discovery (MIND), and the National Science Foundation (NSF) under ECCS Award No. 0925844. All the calculations were performed using computational resources of Texas Advanced Computer Center (TACC) at University of Texas at Austin.

${ }^{1}$ J. A. del Alamo, Nature (London) 479, 317 (2011).

${ }^{2}$ H.-S. Kim, I. Ok, M. Zhang, F. Zhu, S. Park, J. Yum, H. Zhao, J. C. Lee, J. Oh, and P. Majhi, Appl. Phys. Lett. 92, 032907 (2008).

${ }^{3}$ J. Robertson, Microelectron. Eng. 86, 1558-1560 (2009).

${ }^{4}$ W. Wang, G. Lee, M. Huang, R. M. Wallace, and K. J. Cho, J. Appl. Phys. 107, 103720 (2010).

${ }^{5}$ R. V. Galatage, H. Dong, D. M. Zhernokletov, B. Brennan, C. L. Hinkle, R. M. Wallace, and E. M. Vogel, Appl. Phys. Lett. 99, 172901 (2011).

${ }^{6}$ W. Wang, K. Xiong, R. M. Wallace, and K. Cho, J. Phys. Chem. C 114(51), 22610-22618 (2010).

${ }^{7}$ W. Spicer, I. Lindau, P. Skeath, and C. Su, J. Vac. Sci. Technol. 17, 1019 (1980).

${ }^{8}$ J. M. Woodall, G. D. Pettit, T. N. Jackson, C. Lanza, K. L. Kavanaghs, and J. W. Mayer, Phys. Rev. Lett. 51, 1783-1786 (1983).

${ }^{9} \mathrm{C}$. Wilmsen, Physics and Chemistry of III-V Compound Semiconductor Interfaces (Plenum, New York, 1985).

${ }^{10}$ B. Brennan, H. Dong, D. Zhernokletov, J. Kim, and R. M.Wallace, Appl. Phys. Express 4, 125701 (2011).
${ }^{11}$ Y. Guo, L. Lin, and J. Robertson, Appl. Phys. Lett. 102, 091606 (2013).

${ }^{12}$ H.-C. Chin et al., IEEE Electron Device Lett. 32, 146 (2011).

${ }^{13}$ J. J. Gu, Y. Q. Liu, Y. Q. Wu, R. Colby, R. G. Gordon, and P. D. Ye, Tech. Dig. - Int. Electron Devices Meet. 2011, 769.

${ }^{14}$ M. Scarrozza, G. Pourtois, M. Houssa, M. Heyns, and A. Stesmans, Phys. Rev. B 85, 195307 (2012).

${ }^{15}$ T. Scimeca, Y. Watanabe, F. Maeda, R. Berrigan, and M. Oshima, J. Vac. Sci. Technol. B 12, 3090 (1994).

${ }^{16}$ Z. Lu, M. T. Schmidt, R. M. Osgood, W. M. Holber, and D. V. Podlesnik, J. Vac. Sci. Technol. A 9, 1040 (1991).

${ }^{17}$ S. KC, W. Wang, K. Xiong, R. C. Longo, H. Dong, R. M. Wallace, and K. Cho, J. Appl. Phys. 113, 103705 (2013).

${ }^{18}$ K. Xiong, W. Wang, D. M. Zhernokletov, S. KC, R. C. Longo, R. M. Wallace, and K. Cho, Appl. Phys. Lett. 102, 022901 (2013).

${ }^{19} \mathrm{~L}$. Lin and J. Robertson, J. Vac. Sci. Technol. B 30, 04E101 (2012).

${ }^{20}$ M. P. J. Punkkinen et al., Phys. Rev. B 83, 195329 (2011).

${ }^{21}$ Fundamental Aspects of Silicon Oxidation, edited by Y. J. Chabal (Springer, New York, 2001).

${ }^{22}$ A. Pasquarello, M. S. Hybertsen, and R. Car, Nature (London) 396, 58 (1998).

${ }^{23}$ G. Kresse and J. Furthmuller, Comput. Mater. Sci. 6(1), 15-50 (1996).

${ }^{24}$ R. G. Parr and W. Yang, Density-Functional Theory of Atoms and Molecules (Oxford University Press, New York, 1989).

${ }^{25}$ P. Hohenberg and W. Kohn, Phys. Rev. 136, B864 (1964).

${ }^{26}$ W. Kohn and L. J. Sham, Phys. Rev. 140, A1133 (1965).

${ }^{27}$ G. Kresse and D. Joubert, Phys. Rev. B 59, 1758 (1999).

${ }^{28}$ P. E. Blochl, Phys. Rev. B 50, 17953 (1994).

${ }^{29}$ G. Kresse and J. Hafner, Phys. Rev. B 47, 558 (1993).

${ }^{30}$ G. Kresse and J. Furthmuller, Phys. Rev. B 54, 11169 (1996).

${ }^{31}$ G. Kresse and J. Hafner, Phys. Rev. B 49, 14251 (1994).

${ }^{32}$ J. P. Perdew, K. Burke, and M. Ernzerhof, Phys. Rev. Lett. 77, 3865 (1996).

${ }^{33}$ W. Tang, E. Sanville, and G. Henkelman, J. Phys.: Condens. Matter 21, 084204 (2009).

${ }^{34}$ E. Sanville, S. D. Kenny, R. Smith, and G. Henkelman, J. Comput. Chem. 28, 899-908 (2007).

${ }^{35}$ G. Henkelman, A. Arnaldsson, and H. Jónsson, Comput. Mater. Sci. 36, 354-360 (2006).

${ }^{36}$ H. Dong, B. Brennan, D. Zernokletov, J. Kim, C. L. Hinkle, and R. M. Wallace, Appl. Phys. Lett. 102(17), 171602 (2013).

${ }^{37}$ H. Dong, W. Cabrera, R. V. Galatage, S. KC, B. Brennan, X. Qin, S. McDonnell, D. Zhernokletov, C. L. Hinkle, K. Cho, Y. J. Chabal, and R. M. Wallace, Appl. Phys. Lett. 103, 061601 (2013).

${ }^{38}$ O. Ceballos-Sanchez, A. Sanchez-Martinez, M. O. Vazquez-Lepe, T. Duong, R. Arroyave, F. Espinosa-Magana, and A. Herrera-Gomez, J. Appl. Phys. 112, 053527 (2012).

${ }^{39}$ R. M. Wallace, ECS Trans. 16(5), 255-271 (2008).

${ }^{40}$ J. Robertson, Appl. Phys. Lett. 94, 152104 (2009).

${ }^{41}$ P. W. Peacock, K. Xiong, K. Tse, and J. Roberton, Phys. Rev. B 73, 075328 (2006).

${ }^{42}$ Y. F. Dong, Y. P. Feng, S. J. Wang, and A. C. H. Huan, Phys. Rev. B 72, 045327 (2005).

${ }^{43}$ H. Dong, S. KC, X. Qin, B. Brennan, S. McDonnell, D. Zhernokletov, C. L. Hinkle, J. Kim, K. Cho, and R. M. Wallace, J. Appl. Phys. 114, 154105 (2013). 